Ingeborg Jandl*

\title{
Emotionale Objekte und die Last der Vergangenheit
}

\author{
Symbolische Präsenz und physische Verarbeitung \\ transgenerationaler Traumata in der russischen und \\ südslawischen Literatur
}

https://doi.org/10.1515/slaw-2021-0013

Summary: In many literary texts the haptic symbolism of transgenerational trauma is illustrated by objects. Inherited artefacts are in this understanding symbolic as well as indexical: e.g. when amulets, that are worn around the neck, illustrate a burden inherited from earlier generations. Such a meaning becomes often evident already in the symbolic act of passing down an object to a successor. The paper lays its focus especially on motifs of interaction between characters and objects that illustrate their coping with the emotional charge of the past. This is to shed light on the emotional value of different objects and on the mimesis of the physiologically perceived burdens of trauma.

Keywords: objects and narration, mimesis of emotions, transgenerational trauma, traumatic memory

\section{Einleitung: Literarisches Erinnern anhand von Objekten}

Auf die Bedeutung von Erinnerungsobjekten wurde im kulturwissenschaftlichen Kontext u.a. von Aleida Assmann hingewiesen:

\footnotetext{
Zwischen einem Besitzstück und seinem Besitzer besteht ein subtiles Band. Dieses Band wirkt in zwei Richtungen: Die Besitzerin reichert das Besitzstück mit biografischer Bedeutung an, und das Besitzstück wird zur Stütze der Identität der Besitzerin. [...] Die Gegenstände, mit denen wir umgehen und die uns ans Herz gewachsen sind, sind keine toten Objekte, sondern die ebenso intime wie lebendig-pulsierende Peripherie unserer Person. (Assmann 2003: 149)
}

*Kontaktperson: Dr. Ingeborg Jandl, Institut für Slawistik, Universität Wien, Spitalgasse 2, Hof 3, A-1090 Wien, E-Mail: ingeborg.jandl@univie.ac.at 
Diese unmittelbare Verwobenheit mit Menschen bildet die Grundlage für die symbolische Bedeutung, mit der Objekte auch in transgenerationalen Konstellationen familiäre Beziehungsqualitäten illustrieren können.

Bereits Sigmund Freud konstatierte eine „verlangte Kontinuität im Seelenleben der einander ablösenden Generationen“ (Freud 2015: 221) und daran anknüpfend geht es im Folgenden um die transgenerationale Weitergabe von Erinnerungen. Der Psychologe Franz Breuer geht auf eine solche Tradierung im Zusammenhang mit einem quasi „magischen Gebrauch“ von Objekten ein und beschreibt dabei Konstellationen, in denen Fotos, Schmuckstücke und andere Erinnerungsgegenstände „stellvertretend für die andere Person gebraucht werden“ (Breuer 2015: 44).

Diese Beobachtung erlaubt die unmittelbare Verbindung mit Marianne Hirschs Konzept der Postmemory, welches Hirsch hauptsächlich verwendet, um die indirekte Traumatisierung der Nachkriegsgeneration und der Nachfahren von Opfern des Holocaust zu untersuchen. (vgl. Hirsch 2012: 3-4) Obgleich sie den Schwerpunkt auf die Analyse imaginierter Vergangenheiten anhand von Fotografien legt, die sich aufgrund der verborgenen Details im Bildinhalt besonders zur Spurensuche eignen, weist sie gemeinsam mit Leo Spitzer überlieferten Objekten eine ähnliche Rolle zu:

Such 'testimonial objects' carry memory traces from the past, to be sure, but they also embody the very process of its transmission. They testify to the historical contexts and the daily qualities of the past moments in which they were produced and, also, to the ways in which material objects carry memory traces from one generation to the next. (Hirsch \& Spitzer 2012: 178)

Dass Objekte, die mit früheren Lebensabschnitten assoziiert sind, Erzählanlässe bieten, da sie auch schwer zugängliche, traumatisch besetzte Inhalte zu Narrativen bündeln, zeigen u.a. die nicht-literarischen Texte im Sarajevoer ,Museum der Kriegskindheit‘ (Muzej ratnog djetinjstva) (vgl. Jandl 2019b).

Innerhalb der Literaturwissenschaft betreffen Inszenierungen von Erinnerungsobjekten das Forschungsfeld literarischer Gedächtnisdiskurse. Darin verankert liegt u.a. die Forschung zur Erinnerungshaftigkeit von Texten, d.h. die Auseinandersetzung mit erzählerischen Verfahren, die aus der Psychologie bekannte Gedächtnisprozesse quasi-mimetisch nachahmen.

Aleida Assmann illustriert dies u.a. an traumatischen Flashbacks sowie am Zusammenspiel von Erinnerung und Raumwahrnehmung, die sich in der Literatur häufig analog zu realen Erinnerungsprozessen feststellen lassen (vgl. Assmann 2010: 278-280); Michael Basseler und Dorothee Birke weisen auf narrative Verfahren wie Analepsen und iteratives Erzählen hin, die jeweils auf textimmanentes Erinnern verweisen und, etwa bei Proust, anhand von erinnerungsgenerie- 
renden Motiven motiviert werden können (vgl. Basseler \& Birke 2005: 126-129). Prousts Motiv der ,unwillkürlichen Erinnerung', d.h. der Analepse, die auf der Handlungsebene durch ein erinnerungsauslösendes Geruchs- oder Geschmackserleben eingeleitet wird, hat sich unter der Bezeichnung ,Proust-Effekt‘ mittlerweile auch in der Psychologie etabliert (vgl. Goldstein 2015: 380). ${ }^{1}$

Das Erzählen anhand von Erbstücken kann ebenfalls als Verfahren beschrieben werden, das eine spezifische Form von ,Erinnerungshaftigkeit“ motiviert. Ariane Eichenberg stellt in Bezug auf deutschsprachige Generationenromane eine transgenerationale Verknüpfung von Biografien fest, „die schwerlich durch offizielle Archivdokumente hergestellt werden kann, wohl aber durch Gegenstände“ (Eichenberg 2009: 41). Während die ,unwillkürliche Erinnerung‘ weit zurückliegende Ereignisse in einem Moment der Gegenwart plötzlich unmittelbar zugänglich macht, schlagen Erbstücke eher durch ihre Eigenschaft der Beständigkeit zu Buche. Aus diesem Grund sind sie für die Verhandlung transgenerationaler Konstellationen besonders geeignet.

In den anschließend behandelten Beispielen zeichnet sich der Prozess des Erinnerns anhand von vererbten Gegenständen als längerfristige Auseinandersetzung mit einer traumatischen Vergangenheit aus, die erst Stück für Stück erschlossen und in die Gegenwart überführt werden muss. In mehreren Texten markiert jeweils ein Objekt eine bestehende Lücke und verweist symbolisch-indexikalisch auf ein Familiengeheimnis, das es durch mühsame Nachforschungen zu erschließen gilt.

\section{Forschungsfrage und Korpus}

Der Beitrag widmet sich literarischen Narrativen der Weitergabe und des Zurücklassens traumatischer Erfahrungen. Das Hauptaugenmerk liegt dabei auf der Stellvertreter-Rolle von Objekten und konkreter physischer Bewegung in symbolischen Akten der Auseinandersetzung, der Neuverortung und des Abschiednehmens von transgenerationalem Erbe. Die ontologische Perspektive dieser Studie bezieht die Annahme ein, dass diese Motivkomplexe eng mit den psychischen Strukturen von Traumatisierung und Traumaverarbeitung korrelieren, was für eine interdisziplinäre Relevanz der Ergebnisse sprechen würde.

1 Zur Verbindung zwischen Sinneswahrnehmungen und Emotionen als Grundlage der Informationsspeicherung und Auslöser von Erinnerungen auf textimmanenter Ebene in der Literatur siehe außerdem Jandl 2019. 
Im Mittelpunkt der Analyse steht die Konstellation um das titelgebende Möbelstück in Ananij Kokurins Roman Der Tisch (2018), das das Sprechen über die Vergangenheit einleitet und anschließend zum Gegenstand physischer Auseinandersetzung mit einem lange verschwiegenen Familiengeheimnis und einer transgenerational vererbten Traumatisierung wird. Metaphorische Sequenzen und physische Verankerung des Erlebens verschränken sich dabei ineinander. Im vorliegenden Fall wird die Rolle des Tisches durch die ungewöhnliche Handlung eines beschwerlichen Umzuges unterstrichen, bei dem zwei Frauen das sperrige Objekt unter großer körperlicher Anstrengung über eine weite Distanz befördern.

Die hier am metaphorisch-leitmotivischen Tisch festgemachten, physisch verhandelten psychischen Prozesse von Weitergabe, Belastung und Trauma-Arbeit werden durch den Vergleich mit anderen literarischen Beispielen kontrastiert und verortet: Das heterogene Korpus aus Narrativen, die sich auf russische und südslawische Handlungsräume beziehen und in mehreren Fällen zusätzlich Räume der Migration nach Mitteleuropa oder in die USA einschließen, wurde auf Basis struktureller Überlegungen gewählt. Da Andenkenstücke in erzählerischen Auseinandersetzungen mit transgenerationalen Traumata zum Standard-Repertoire zählen, könnte es relativ einfach durch weitere Texte aus diesen und anderen Kulturräumen ergänzt werden.

Im Zentrum der Analyse steht nicht die jeweilige Handlung über geschichtlich und kulturell verortete individuelle Traumata, sondern das strukturelle Geflecht von deren Darstellung unter Rückgriff auf Gegenstände, die die Vergangenheit leitmotivisch im Gesamttext konservieren. Dabei wird davon ausgegangen, dass diese Objekte jeweils eine nicht generalisierbare konkrete Dynamik entfalten, die das betreffende Narrativ unterstützt, dass sie jedoch zugleich per se, aufgrund ihrer Materialität und Funktion als Gebrauchsgegenstände, bereits eine spezifische Dynamik implizieren. Beide Komponenten sollen Berücksichtigung finden. Während der erwähnte Küchentisch den Blick auf den Schauplatz des geschützten Innenraums von Küche und Esszimmer lenkt - wo die verschwiegenen Ereignisse hauptsächlich zu verorten sind -, wird er im Rahmen dieses TraumaTexts v.a. durch seine Größe und Sperrigkeit für die Darstellung physisch-emotionaler Grenzerfahrung produktiv gemacht.

Eine direktere Symbolik in Hinblick auf die transgenerationale Weitergabe einer Bürde haftet hingegen Amuletten an; eine damit verbundene Motivik des Annehmens und Ablegens von Belastung wird am Beispiel der Texte von Petr Aleškovskij, Danilo Kiš und Sergej Lebedev aufgezeigt. Auf komplementäre Weise gilt Ähnliches für das mit dem Film Grbavica in die Überlegungen integrierte Motiv der Waffe, das den zurückliegenden gewaltbedingten Verlust als konkrete Gefahr in die gegenwärtigen Auseinandersetzungen mit dem Trauma trägt und damit auch die Hilflosigkeit gegenüber Vergangenem illustriert. Zur Kontrastierung 
des beschwerlichen Weges, der in Kokurins Roman die Trauma-Arbeit erfahrbar macht, dient schließlich ein Roman von Il'ja Bojašov, in dem das Trauma ohne Umstände zurückgelassen wird. Als zwei kanonische Texte ergänzen dieses Korpus zusätzlich Sergej Dovlatovs Čemodan, der das Motiv des transgenerationalen Erzählens anhand von Gegenständen als strukturgebend fruchtbar macht, sowie (als einziger Text, der nicht den slawischen Literaturen entstammt) Wolfgang Borcherts Küchenuhr, in der Trauma und individuelle Trauma-Arbeit anhand der komplexen gegenseitigen Spiegelung von Figur und Ding thematisiert werden.

Aufgrund ihrer materiellen Form wird die symbolische Belastung durch Erinnerungen in den gewählten Beispielen einerseits physisch greifbar, andererseits bleibt sie als nonverbale Information für Außenstehende zunächst unzugänglich. Ob und in welcher Form Trauma-Arbeit stattfindet und gelingt, soll im Folgenden am Umgang mit Erinnerungsobjekten untersucht und demonstriert werden, wobei eine grundlegende Frage darin besteht, ob und wie die Erinnerungen verbalisiert werden (können).

\section{Familienerbe und Weitergabe: Glücksbringer und belastende Amulette}

Dass wiederaufgefundene Gegenstände einen Redeanlass bieten, Episoden aus der persönlichen Vergangenheit an die jüngere Generation weiterzugeben und dieser damit ihre Wurzeln zu vermitteln, bildet die klassische Struktur einer Rahmenhandlung etwa in Sergej Dovlatovs Erzählsammlung Čemodan (1986, Der Koffer). Die für Außenstehende hermetisch verschlossene Vergangenheit der alten Kleidungsstücke erweist sich als „intime wie lebendig-pulsierende Peripherie“ des Ich-Erzählers, der seinem Sohn an jedem der mittlerweile nutzlos gewordenen Gegenstände eine persönliche Geschichte aus der Zeit ihrer Verwendung erzählt.

Während die bei Dovlatov aufgefundenen Objekte speziell für Erinnerungen an die Sowjetunion stehen, die eine geografisch-zeitliche Gegenwelt zum Alltag der mittlerweile in den USA lebenden Familie bilden, in den sie nicht reintegriert werden, verbinden andere Erbstücke generationenübergreifende Familiengeschichten. (vgl. Dovlatov 2010: 347-350)

So ist etwa Sergej Lebedevs Reflektorfigur Kirill in Gus' Fric (2018, Kronos' Kinder) im Besitz eines wertvollen Smaragdcolliers, welches seine Großmutter Lina einst von ihrer Mutter erhielt. Es handelt sich um das Verlobungsgeschenk von Karolinas Mutter, dem diese bis zum Tod ihres Mannes schützende Kraft zuschrieb. Der Roman thematisiert wiederholt die Weitergabe dieses Gegenstands, wobei das Augenmerk jeweils auf dem gegenständlichen Wert des Objekts und 
seiner begehrlich betrachteten Schönheit liegt; besonders hervorgehoben wird jedoch seine Funktion des Talismans, die in allen Episoden subtil mit tatsächlich eingetroffenem Unheil zu enden scheint. Jede Generation setzt ihr Vertrauen in den schönen Gegenstand, und jedes Mal erweist sich dieser Glaube als eitle Blendung: Sof'jas Ehemann kommt im Japanischen Krieg um, und ihre Tochter Lina überlebt als einziges Familienmitglied die Petersburger Blockade.

Das Collier ist schöner Gegenstand und Bürde zugleich, und die physische Art seiner Verwendung als Schmuckstück, das um den Hals getragen wird, unterstreicht bei Lebedev beide Bedeutungen. Hierdurch tritt außerdem das Moment der Weitergabe besonders in den Vordergrund, da Glanz und Last von Mutter zu Tochter weitergegeben werden.

И только накануне отъезда мать велела открыть секретный ящичек в комоде [...], достать изумрудное ожерелье, последнее сокровище, о котором сестры в девичестве ревниво спорили, кому оно достанется. Повинуясь матери, Каролина надела ожерелье; мать смотрела на нее, будто наряжала на первый бал; а потом отвернулась и кротко заснула. Каролине показалось, что она видит сновидение матери, вечно живущее в лукавых зеленых [...]. (Lebedev 2018: 335)

Erst am Vortag der Abreise hieß ihre Mutter sie das Geheimfach der Kommode öffnen [...], um das Smaragdcollier hervorzuholen, der letzte Schatz, um den sich die Schwestern in ihren Mädchenjahren eifersüchtig gestritten hatten: wer es erben würde. Karolina gehorchte der Mutter und legte die Halskette an; ihre Mutter betrachtete sie, als habe sie sich für ihren ersten Ball herausgeputzt. Dann drehte sie sich um, und schlief sanft ein. Karolina kam es so vor, als sähe sie die Traumerscheinung ihrer Mutter, die für immer in den tückischen grünen Steinen eingeschlossen war [...]. (Lebedew 2018: 322)

Wie bereits Sof'ja, trägt Lina das lang ersehnte Collier schließlich nur kurze Zeit; sie spürt ihre Familiengeschichte auf sich lasten - den Krieg, die Schifffahrt nach Japan und die Reise über Sibirien - und kann sich, mit diesem Schmuckstück um den Hals, nicht von der darin konservierten Vergangenheit abgrenzen. Kirills Überlegungen kreisen ebenfalls um diese Familienzusammenhänge und um die symbolischen Bedeutungen, die sich in dem Objekt verbinden: die Tode vieler und das Überleben Ausgewählter. Auch Kirill vollzieht seine Abgrenzung physisch anhand dieses Gegenstands: Er verstaut ihn außer Reichweite.

Während die Gegenstände in Dovlatovs Čemodan zum Sprechen anregen und eine Vergangenheit vergegenständlichen, die für die Familie überwunden erscheint und nun als Erinnerung weitergegeben wird, steht das Smaragdcollier in Lebedevs Gus' Fric für belastende Ereignisse, die Kirills Familiengeschichte für immer eingeschrieben erscheinen. Es vergegenwärtigt traumatische Ereignisse, für die in den Sequenzen der Weitergabe keine Worte gefunden werden. In seiner objekthaften, stummen Form werden diese unverarbeitet auf die Folgegenerationen übertragen. 
Beiläufig wird erwähnt, dass das Smaragdcollier die ganze Familie vor dem Petersburger Hunger hätte retten können, wenn man es verkauft hätte. Obgleich dies in der Romanhandlung durch seine räumliche Entfernung während dieser Episode, den Aufbewahrungsort Moskau, mitbestimmt wird, ist der Nicht-Verkauf des für alle aufeinanderfolgenden Besitzer ambivalenten Amuletts auch symbolisch von Interesse. Das Festhalten der Folgegenerationen am Familientrauma wird am Umgang mit diesem Gegenstand nämlich ebenso deutlich, wie die Weitergabe unverarbeiteter Belastungen durch die Eltern.

Eine ähnliche Konstellation der Weitergabe wird in Petr Aleškovskijs Roman Vladimir Čigrincev (1995, Der Erbe) anders gelöst: Tochter Tat'jana verkauft das von ihrem Vater erhaltene wertvolle Collier später, um von dem Erlös die Medikamente und Schmiergelder für die Behandlung von dessen Krebserkrankung zu bezahlen. Die Szene, in der sie das Collier von ihrem Hals nehmen lässt, bildet das komplementäre Gegenstück zu der zuvor aus Lebedevs Roman zitierten, in der die Urgroßmutter der Großmutter das Erbstück vermacht.

Nachdem Aleškovskijs Tat'jana sich das ihr vererbte Collier ebenfalls zum ersten Mal um den Hals gelegt hat, um sich in einem Abendkleid aus schwarzem Samt herausgeputzt von ihrem Verehrer bewundern zu lassen, entledigt sie sich dieser Familienbürde: der trüben Erinnerung an ihre vom dominanten Vater beherrschte Kindheit ebenso, wie dessen Obsession nach wertvollem alten Schmuck.

- [Р]асстегни, пожалуйста, колье, - она повернулась спиной, - там такой гвоздик хитрый. Пришлось повозиться - замок был простой, но очень тугой, добротный. Татьяна терпеливо ждала. Наконец цепь неограненных рубинов распалась [...]. - [...] [Э]то камни папины, всю жизнь он за них цеплялся [...]. Разве ты не понял? [...] Я же жить тем, чего не видала и не знаю, не хочу и не буду. Понял? (Aleškovskij 1997: 74-75)

„[...] Mach mir bitte mal das Kollier auf.“ Sie drehte ihm den Rücken zu. „Da ist so ein raffinierter Haken. Er brauchte eine Weile“ - es war ein einfacher, aber solide gearbeiteter Verschluss. Tatjana wartete geduldig. Endlich löste sich die Kette [...]. „[...] [D]as [sind] Papas Steine, sein Leben lang hat er sich an sie geklammert [...]. Hast du das nicht begriffen? [...] Was ich nicht aus eigener Anschauung kenne und erfahren habe, das wird in meinem Leben keinen Platz haben. Klar?““ (Aleschkowski 1998: 76-77)

Tat'jana vollzieht ihre Entscheidung, ohne den Vater einzuweihen und mit der dezidierten Absicht, das Geld nicht für sich selbst zu nutzen, um die Bürde zurückzulassen. Sie trennt sich physisch von dem, was sein Leben irrational bestimmte und von ihm, ebenfalls physisch, an sie übergeben wurde. 


\section{Amulett, Tisch und Uhr als Spuren der Vergangenheit}

Zugleich verhalten sich verschiedene Objekte aufgrund ihrer Materialität unterschiedlich in ihrer Funktion als Erinnerungsgegenstände. Angesichts ihrer geringeren Haltbarkeit hinsichtlich ihrer Verwendbarkeit über mehrere Generationen hinweg, eignet sich die bei Dovlatov wiedergefundene alte Kleidung ${ }^{2}$ zwar dazu, eigene Erfahrungen zu speichern und autobiografische Erinnerungen für das Erzählen wachzurufen; sie kann aber nicht die Erlebnisse mehrerer Generationen als materielles Objekt speichern und verbinden.

Anders dagegen ist wertvoller Familienschmuck als klassisches Erbstück auch zur späteren Erfahrung transgenerationaler Verbindungen geradezu prädestiniert, zumal er indexikalisch mit den Erlebnissen früher Generationen verbunden bleibt. In den Romanen von Lebedev und Aleškovskij verfestigt sich diese indexikalische Verbindung jeweils zu einem festen Symbol. Dass beide Romane die transgenerationale Weitergabe von Belastung gerade anhand von Colliers symbolisieren, scheint nicht von ungefähr. Das Tragen dieser relativ schweren schmückenden Gegenstände impliziert eine Berührung mit der fragilen Halsregion der Trägerin, wo auch Medizin und Psychosomatik eine sensible Schnittstelle zwischen sinnlicher und emotionaler Wahrnehmung annehmen, die etwa beim Angstempfinden relevant werden kann: „In Analogie zur Mimik gibt es [...] ein Ausdrucksverhalten der Gesamtmuskulatur, besonders der proximal gelegenen tonischen Halsmuskulatur, die die aktuelle psychische Verfassung, aber auch eine seelische Grundhaltung widerspiegelt.“ (Volc, Tilscher \& Hanna 1988: 75)

Zumindest Aleškovskij wählt nicht zufällig dieses eine Collier für die Illustration der Bürde, denn Tat'jana erbte zusätzlich zu dem Collier einen wertvollen alten Ring und ihre Schwester Ol'ga eine Brosche; beide werden jedoch nach ihrer Übergabe weder verwendet noch weiter thematisiert.

2 Neben Kleidungsstücken finden sich außerdem ein Zeitungsausschnitt mit Karl Marx am Boden des Koffers, sowie einer mit Iosif Brodskij an der Deckwand: „Я оглядел пустой чемодан. На дне Карл Маркс. На крышке - Бродский. А между ними - пропащая, бесценная, единственная жизнь. “ (vgl. Dovlatov 2010: 350) (Ich schaute in den leeren Koffer. Am Boden Karl Marx. Auf der Deckenwand Brodskij. Und zwischen ihnen das unrettbare, kostbare, einzige Leben.) Anders als die Kleidungsstücke haben diese Ausschnitte zusätzlich zu ihrer subjektiven indexikalischen Bedeutung durch ihren Bezug zum Ich-Erzähler eine konkretere, auch für Außenstehende sichtbare, kulturelle Bedeutung; wie dieser Kontext zeigt, werden die Ausschnitte im Reflexionsprozess des Ich-Erzählers ebenfalls als indexikalisch für die Lebenswelt der Sowjetunion gedeutet. 
Auch Gebrauchsgegenständen wird auf Basis ihrer indexikalischen Verbindung zur Vergangenheit mitunter im Bewusstsein literarischer Figuren symbolische Bedeutung zugewiesen, wodurch sie zu wichtigen Elementen der Handlung werden können. So kann die Küchenuhr in Wolfgang Borcherts gleichnamiger Dingerzählung von 1947 als Symbol für den posttraumatischen Zustand des Protagonisten selbst nach der Zerstörung seines Elternhauses gelesen werden. Innerlich zerrüttet, lässt seine zwar traumatisch gealterte äußere Erscheinung noch die Bezüge zu seinem früheren Ich erkennen.

Die Zeiger sind natürlich nur aus Blech. Und nun gehen sie auch nicht mehr. Nein. Innerlich ist sie kaputt, das steht fest. Aber sie sieht noch aus wie immer. Auch wenn sie nicht mehr geht. Er machte mit der Fingerspitze einen vorsichtigen Kreis auf dem Rand der Telleruhr entlang. Und er sagte leise: und sie ist übriggeblieben. (Borchert 1999: 103)

Während die Uhr am Beginn symbolisch den Reflexionsprozess über das Selbst nach dem erlittenen Trauma zu begleiten scheint, wird sie später in ein liebevolles Symbol für die Fürsorge der getöteten Mutter umgedeutet; der Protagonist liest die nun unveränderlich angezeigte Uhrzeit als Verweis auf die nächtlich von ihr für ihn aufgewärmten Mahlzeiten. Durch diese kausal nicht motivierte symbolische Umdeutung, die auch die medizinische Perspektive als Ansatz zur TraumaHeilung vorschlägt (vgl. Levine 2016: 177), gelingt dem Protagonisten die emotionale Linderung des Traumas.

Ähnliche Prozesse sind auch strukturgebend für den bislang nur in deutscher Übersetzung vorliegenden Roman Der Tisch (2018, Stol) des russischen Emigrationsschriftstellers Ananij Kokurin. Der titelgebende Tisch markiert hier transgenerationale Verbindungen und verleiht der Preisgabe persönlich-biografischer Informationen eine physische Form. Dass er für die in einer russischen Provinzstadt lebende Mutter der Ich-Erzählerin seit Jahrzehnten als schweigender Zeuge die Vergangenheit bewahrt, wird erst deutlich, als sie sich beim Umzug weigert, den Tisch zurückzulassen. Ein von Aleida Assmann beschriebener Mechanismus der Mensch-Objekt-Beziehung kommt hier zum Tragen: „Spürbar wird es [dieses magische Verhältnis gegenseitiger Aufwertung und Bestimmung von Ding und Person] erst, wenn das Band gekappt wird, wenn man etwas verliert, womit man, wie man dann feststellt, verwachsen war.“(Assmann 2003: 149)

Über den Roman hinweg markiert dieser Tisch außerdem den Chronotopos, denn er illustriert symbolisch und physisch die Befindlichkeit von Menschen und deren situative Verortung in ihren Lebenswegen. Die Chronotopik des Tisches verändert sich; zunächst scheint mehrmals gerade dieser Tisch den Umzug der Mutter zu vereiteln. Der Tisch ist zu groß, um aus der Wohnung gebracht zu werden, sodass man schließlich die Beine absägt, und am Bahnhof angekommen, ist er aufgrund seiner ungewöhnlichen Form als Gepäcksstück in der Eisenbahn 
nicht erlaubt, sodass sich Mutter und Tochter dazu entschließen, den weiten Weg zu Fuß zurückzulegen.

Der stumme Widerstand dieses Objekts bleibt jedoch beide Male nicht ohne Folgen, sondern bietet im ersten Fall den wichtigen Anlass zu einem ersten Gespräch über die der Tochter unbekannte Vergangenheit der Mutter. Der Tisch wird im Zuge dieser langen Erzählung außerdem ein letztes Mal vor dem Umzug in seiner eigentlichen Funktion als Esstisch benutzt. Im zweiten Fall bedeutet die plötzliche Notwendigkeit, zu Fuß zu gehen, dass der Weg länger und beschwerlicher wird; der Tisch bildet hier abermals ein Moment der Verzögerung, das die Beteiligten zur Auseinandersetzung mit der Familien-Vergangenheit zwingt.

\section{Tisch und Fotografie: Zwei unterschiedliche Materialitäten der Erinnerung}

In Kokurins Tisch wird die unterschiedliche Dynamik, die Gegenstände aufgrund ihrer verschiedenartigen Materialität in ihrer Funktion als Erinnerungsobjekte entfalten können, besonders deutlich. Das Kernelement des Familiengeheimnisses stellt hier die lang zurückliegende und bislang verschwiegene Beziehung der Mutter zu einem Wehrmachtssoldaten dar. Die Mutter legt diese Verbindung zunächst nicht erzählerisch, sondern anhand eines Fotos offen, das sie aus einem unter die Tischplatte genagelten Brett hervorholt: „Meine Mutter hielt ein Foto in der Hand. Das unerträgliche Foto, auf dem sie und der junge Mann in der deutschen Uniform so albern posierten, während sie einander umarmten.“ (Kokurin 2018: 34)

Die Spuren dieses Mannes verlieren sich nach dem Krieg. Bei der Protagonistin führen die Erzählungen der Mutter schließlich zu einer Umwertung dieser zunächst, wie an dem Zitat deutlich wurde, abgelehnten Verbindung. Die nachträglichen Informationen über Georg veranlassen jedoch dann sogar eine Restrukturierung der eigenen, sicher geglaubten Kindheitserinnerungen, die gar nicht auf den leiblichen Vater, sondern auf diesen anderen Mann verweisen:

Daher kamen also die Stimmen, die ich gehört hatte! Stimmen, die mich das ganze Leben hindurch begleiteten! Plötzlich sah ich alles klar vor mir - mein Gott, ich war ja gerade ein Jahr alt, als mein Vater an die Front eingezogen wurde. Wie sollte ich mich an ihn erinnern? Es war ganz klar: nicht an seine Hände erinnerte ich mich so oft, nicht meines Vaters Stimme, nicht sein Lachen hörte ich, wenn ich versuchte, ihn mir vorzustellen. Jener Mann aus meiner Kindheit war Georg von Gotthardt, ein deutscher Offizier, der meine Mutter liebte und mir als Kleinkind das Leben rettete. (ebd. 56) 
Durch das Erkennen eines Zusammenhangs mit den eigenen verschwommen frühkindlichen Erinnerungen wird die familiäre Verbindung zu Georg, der den besagten Esstisch gezimmert hat, zu einer innigen emotionalen Bindung an eine Vaterfigur. Anhand dieser Geschichte wird außerdem der seit der Kindheit bekannte unbedeutende Tisch neu in die Biografie der Protagonistin integriert, die den Wunsch der Mutter, das schwere Objekt nach Nižnij Novgorod mitzuübersiedeln, nun unterstützen will: „Denn eins war mir klar - der Tisch meiner Mutter gehörte in Zukunft in mein Leben.“ (ebd. 81)

An dieser Stelle bietet sich der Vergleich zwischen den Erinnerungsobjekten Fotografie und Tisch an, denn man könnte die Frage stellen, warum in diesem Roman der Tisch so stark im Vordergrund steht, obwohl der Autor Fotograf ist und obwohl die Fotografie so viel konkreter zu sein scheint in ihrem Potenzial, Bezüge zwischen Vergangenheit und Gegenwart herzustellen. Wie anhand der oben thematisierten Fotografie deutlich wurde, lösen Bilder von Georg ambivalente Assoziationen aus, weil er auf ihnen visuell so deutlich als Offizier der Wehrmacht zu erkennen ist. Dieser Umstand kann aus der visuellen Einheit des Fotos nicht ausgeblendet werden. Der Tisch dagegen stellt einen belanglosen Gegenstand aus der Kindheit dar, der nun zwar mit Georg als seinem Urheber assoziiert wird, jedoch primär auf jenen privaten Kontext bezogen bleibt, in dem diese Urheberschaft stattfand. Außerdem enthält er keine indexikalischen Verweise auf politische Konstellationen, und bleibt so, massiv und behäbig, ein rein privates Objekt und Konstrukt. Als solches vereint er nun für alle, die über öffentlich verschwiegene Episoden Bescheid wissen, die Familienerinnerung.

\section{Tisch, Trauma, Traumaverarbeitung}

Anhand des Tisches wird außerdem die subjektive Prägung belastender Familienerinnerung deutlich: Das Tragen und Schieben eines massiven Tisches, der aufgrund seines Werts als Erinnerungsobjekt nicht zurückgelassen wird, illustriert die physische Anstrengung, die im Prozess des Nacherinnerns aufgewendet wird. Aufgrund der physikalischen Eigenschaften des Tisches ist die Reise beschwerlich, weshalb auf unterschiedlichen Etappen des Weges auch längerfristig geschwiegen wird. Außerdem beginnt die Mutter weiterführende Fragen über Georg und die Vergangenheit absichtlich zu überhören. Der Tisch und das Zurücklegen der Wegstrecke treten hier an die Stelle des Erzählens. Diesem Kontext entstammt auch die folgende Textstelle:

Tisch, Tisch, Tisch, Tisch, Tisch, Tisch, Tisch, Tisch, Tisch, Tisch, Tisch, Tisch, Tisch, Tisch, Tisch, Tisch, Tisch, Tisch, Kanaille, Tisch, Tisch, Tisch, Tisch, Tisch, Tisch, Tisch, Tisch, Tisch, 
Tisch, Tisch, Tisch, Tisch, Tisch, Tisch, Tisch, Tisch, Tisch, Tisch, Tisch, Tisch, Tisch, Tisch, Tisch, Tisch, Tisch, Tisch, Tisch, Tisch, Tisch, Tisch, Tisch, Tisch, Tisch, Kanaille (ebd. 108)

Man könnte diese formal auffällige Episode als Illustration der Gedanken und der emotionalen Situation der beiden Frauen verstehen, die in die durch den Tisch gebündelte Geschichte völlig versunken sind. Es ist eine Art physischer Präsenzerfahrung des Tisches, der mit dem ganzen Körper getragen und angenommen wird, womit die Protagonistinnen sich zugleich physisch und emotional auf eine für sie selbst unkontrollierbare Situation eingelassen haben. Durch die schweigende Kommunikation kommen sie ihrer Vergangenheit so nahe, wie physisch dem Tisch. Diese Tisch-Erfahrung ist nicht wie eine Erzählung strukturiert, die Veränderungen und Bewertungen implizieren würde. Sie gleicht der reinen physischen Präsenzerfahrung des Tragens, die hier visuell und non-verbal verdeutlicht wird, als beschwerlich, zirkular sowie damit außerdem als unveränderlich, stagnierend und womöglich unverständlich. Peripher mischen sich daher auch Flüche in diesen an sich höchst abstrakt bleibenden „Tisch“-Gedanken. Eingeleitet wird die Episode, wie folgt, physikalisch: „Die Hypothese von der doppelten Seele besagt, dass ,sich jede beliebige kompakte Mannigfaltigkeit einer nichtnegativen Tiefkrümmung mit zwei Schichten bedecken lässt'.“ (ebd.) Dies ist ein vergleichsweise schwer verständlicher Gedanke in diesem Roman, der damit auch als metasprachlicher Ausdruck der schweren Verständlichkeit des „Tisch“-Gedankens interpretiert werden kann. Die beiden Schichten der doppelten Seele sind hier offenbar der unendliche Gedanke an den „Tisch“ mit all seinen Implikationen sowie der auf die ungemütliche Erfahrung bezogene unendliche Gedanke „Kanaille“. Der letztere bleibt auf Basis seiner geringeren Quantität offenbar diskret im Hintergrund, andererseits wird er jedoch auch exponiert, als am Ende der Rhythmus durchbrochen wird, und der Fluch zudem kursiv hervorgehoben ist.

Das Gedicht scheint vor allem die Gedanken der Ich-Erzählerin wiederzugeben, die noch damit beschäftigt ist, die neuen Informationen zu ordnen, während ihre Mutter, nun, nachdem sie ihr Geheimnis geteilt hat, gelassen wirkt: „Obwohl sie jetzt hinkte, hin und wieder tief Atem holte und langsamer ging, ließ meine Mutter weder Unzufriedenheit noch den Wunsch erkennen, vor Sturm und Unwetter Schutz zu suchen. Mein Geschimpfe richtete sich also nur gegen mich selbst.“ (ebd. 109) Gedicht und Unwetter markieren einen Umbruch, der die Ich-Erzählerin aus dem überwältigenden Tisch-Gedanken reißt.

Im Laufe der Reise schafft auch sie die Verarbeitung der gemeinsamen Traumata und es heißt: „Das Gefühl einer ungewöhnlichen Leichtigkeit erfüllte uns.“ (ebd. 161) Der Weg wird auch tatsächlich leichter, als die Frauen per Autostopp im Lastwagen weiterreisen und am Ende verpufft selbst der Reisemodus, da sie vor der Weiterreise nach Nižnij Novgorod eine Woche in der Luxussuite eines 
Hotels direkt am Roten Platz verbringen, wo sie sich neue Frisuren und Kleider zulegen und das durch den Hausverkauf erworbene Geld verprassen. Alles, was belastet hat, wird aufgebraucht und zurückgelassen, wie schon bei Aleškovskij, wo Tat'jana nach dem Verkauf des Colliers das Geld großzügig an die behandelnden Ärzte verteilt.

Kokurins Figuren behalten den Tisch vorerst noch, doch wie aus der Rahmenerzählung bekannt ist, wird auch er nach unbestimmter Zeit weggegeben werden. Dass ein wochenlang zu Fuß übersiedelter Tisch bereits nach wenigen Jahren weggegeben wird, verdeutlicht, dass die wichtigste Funktion dieses Tisches in der Traumaverarbeitung liegt, wo er als Objekt fungiert, das eine physische Verbindung zur Vergangenheit herstellt und einen Reibungspunkt mit dieser erzeugt.

\section{Das Zurücklegen von Wegen als physische Verarbeitung von Erinnerung}

So positiv der Tisch die ganze Handlung über thematisiert wird, so sehr steht doch gerade er auch immer im Weg, behindert er doch die regulären Abläufe eines gewöhnlichen Umzugs. Um das Trauma zurückzulassen, ist daher auch eine Loslösung vom Tisch und der daran gekoppelten Vergangenheit notwendig. Sowohl physisch als auch emotional scheint dieser Prozess der Loslösung erfolgreich verlaufen zu sein, denn bereits vorab in die Schilderung der Reise mit dem Tisch integriert, bemerkt das erzählende Ich: „Wie sehr ich mich auch anstrenge, mich an bestimmte Einzelheiten unserer Reise zu erinnern, sie sind wie ausgelöscht ins Nichts versunken.“ (ebd. 100) Als erschwerendes Element vollzieht der Tisch so die Belastung physisch mit und seine zugleich sinnlich-spürbare und metaphorische Bedeutung moderiert auch die Auflösung des transgenerationalen Traumas durch das Zurücklassen des Tischs.

Im Gegensatz dazu kommt es in Danilo Kišs erstem Roman Psalam 44 (1962, Psalm 44) in Hinblick auf die längerfristig in einem Sarg versteckt liegende Jüdin Aniela zu einem Chronotopos der Bewegungslosigkeit:

Onda Marija zaustavi svoj pogled na ogrlici sa krupnim veštačkim zrnima boje ametista: ta je ogrlica na tankom vratu lutke-spavača davala Anijeli izgled onih mumija što ih nalaze u piramidama faraona: dostojanstvo vladarske smrti. „To je od pokojne mame“ [...]. (Kiš 2006: 31-32)

Dann blieb Marias Blick an einer Kette mit großen amethystfarbenen Kunstperlen hängen: Diese Kette am dünnen Hals der Schlafpuppe verlieht Aniela das Aussehen der Mumien, 
die man in den Pyramiden der Pharaonen findet: die Würde des Herrschertodes. „Die habe ich von meiner verstorbenen Mama.“ (Kiš 2019: 32)

Dass Aniela die amethystfarbene Kette um ihren Hals von ihrer Mutter erbte, bedeutet, dass diese insbesondere auch für die mütterlicherseits vererbte Zugehörigkeit zum Judentum steht und dadurch mit der Bürde eines Lebens im Sarg während der Shoah verknüpft ist. Die absolute Reduktion von Wegen auf nächtliche Toilettenbesuche und der tagelange Rückzug in die durch Schlafmittel und Dunkelheit gedämpfte Anderswelt werden so auch in diesem Familienerbstück reflektiert, das nicht abgestreift werden kann. Indem die Kette zudem an einen Rosenkranz erinnert, wird auch die symbolische Verbindung zu den religiösen Hintergründen der ausweglosen Situation offenbar.

Zugleich ist die Perlenkette das Einzige, was der Figur bleibt und an das sie sich in ihrem dämmrigen Bewusstseinszustand klammert. Als Kalender und imaginäres Musikinstrument spendet sie Aniela Halt, Orientierung und ästhetische Imagination, womit sie ihr dabei hilft, auszuharren.

„Ovo mi služi kao kalendar“, reče ona. „Ako se svako zrno uzme kao jedan mesec, onda tu ima preko tri godine. Rat će se valjda dotle završiti. A može se uzeti i kao nedelja. Ili kao dan. Ili sat.“ [...] „I sviram često kada mi je dosadno“, reče mumija a njeni prsti boje tamnog ćilibara hitro preleteše po ogrlici, zatim zastadoše na tren kao da su našli pravi ton i akord, za sve njih nečujan, ali koji se video u njenim očima preko kojih su preletale lake senke neke nezemaljske melodije [...]. (Kiš 2006: 32-33)

„Das dient mir als Kalender“, sagte sie. „Wenn jede Perle für einen Monat steht, sind das über drei Jahre. Bis dahin wird der Krieg wohl vorbei sein. Aber sie kann auch für eine Woche stehen. Oder für einen Tag. Oder eine Stunde.“ [...] „Ich musiziere oft, wenn es mir langweilig ist“, hatte die Mumie gesagt, und ihre Finger von der Farbe dunklen Bernsteins waren flink über die Kette geglitten, hatten dann einen Moment innegehalten, als hätten sie den richtigen Ton und Akkord getroffen, unhörbar für sie alle, aber sichtbar in ihren Augen [...]. (Kiš 2019: 33)

Der absolute Rückzug in die durch Schlafmittel gestützte Bewegungslosigkeit in der Dunkelheit eines Sarges zeigt dennoch, dass dieser Halt durch die Kette nur ein imaginärer sein kann, der ihr dabei hilft, zu verdrängen, dass die äußere Situation sich in einer Endstation verliert. Die durch Mikrobewegungen der Finger bekräftigte Kondensation des verblassenden Bewusstseins und Selbstgefühls in die geerbte Kette markiert so den Endpunkt einer Familiengeschichte, aus der es kein Entkommen gibt und die auch nicht verarbeitet werden kann.

Ein anderes Beispiel, in dem die chronotopische Bedeutung des Gehens an die Empfindung des Tragens von Gepäck gekoppelt ist, welches sowohl physisch-konkret als auch metaphorisch für Belastung steht, ist Il'ja Bojašovs Roman 
Put' Muri (2007, Muris Weg). Hier wird im Gegenteil die Leichtigkeit des Gehens ohne Gepäck symbolisch wirksam gemacht, zur Illustration des mühelosen Zurücklassens einer Katastrophe. Die Reflektorfigur ist hier ein Kater, der sich nach Ausbruch des Bosnienkrieges aus einem zerstörten Haus und aus Sarajevo entfernt. Seine Reise nach Mitteleuropa wird wiederholt als schwerelos charakterisiert. Gerade darin kontrastiert sie mit jener der zahlreichen menschlichen Nebenfiguren, die nicht nur durch die ausgewählten Habseligkeiten in ihrem Gepäck belastet werden, sondern auch Verluste und Zerstörung beklagen, was noch viel schwerer wiegt. Gleichwohl durch die Detonationen erschreckt, legt der Kater seinen Weg geradezu gleichgültig zurück, ohne Wehmut für seinen früheren Wohnort oder die getötete Familie und ohne Mitgefühl oder Mitleid für die traumatisierten Menschen, denen er sich jeweils nur kurzfristig als distanzierter Reisegeselle anschließt.

А кот трусил по дороге, уводящей из сожженной деревни к весьма недалекой отсюда боснийской столице. Проезжая мимо него, нещадно чадили трубами тракторы с беженцами; их дым отравлял и небо, и землю. С прицепов доносились женские вопли и детский плач. Мужчины от подобных криков зажимали уши. Мури не утруждал себя бесполезной сентиментальностью - его сердце работало без всякого волнения, легкие вполне справлялись с тяжелым воздухом, лапы послушно пружинили. (Воjašov 2011: 29)

Der Kater trottete die Straße entlang, die aus dem niedergebrannten Dorf in die nahe bosnische Hauptstadt führte. Gnadenlos rußten vorbeifahrende, mit Flüchtlingen beladene Traktoren aus ihren Rohren; der Qualm vergiftete Himmel und Erde. Aus den Anhängern drang das Jammern von Frauen und Kinderweinen. Die Männer hielten sich die Ohren zu. Muri belastete sich nicht mit nutzloser Sentimentalität - sein Herz schlug ohne jede Aufregung, die Lungen kamen mit der schweren Luft ganz gut zurecht, die Pfoten federten gehorsam. (Bojaschow 2018: 23)

Der postkatastrophische Zustand des Katers unterscheidet sich nicht von seinem früheren, was als Indikator dafür gelesen werden kann, dass keine Traumatisierung vorliegt. Seine Resilienz erklärt sich aus dem Fehlen von Bindung, die weder in Hinblick auf Menschen noch auf andere Tiere oder materielle Objekte vorliegt. Die Erschütterung betrifft ihn daher nur temporär im Kontext lebensgefährlicher Gefahr; da er ohne Wehmut alles zurücklassen kann, hinterlässt sie keine dauerhafte Spur. 


\section{Fehlen eines Objekts, Verschwinden einer Geschichte}

Wie die Chronotopik von Muris Reise in Bojašovs Roman zeigt, kann auch die Abwesenheit von Objekten in Zusammenhang mit der Verarbeitung von Erlebnissen stehen; im vorliegenden Fall bedeutet sie, dass schmerzhafte Erfahrungen vollständig zurückgelassen werden.

Dass ein abwesendes Objekt transgenerationale Erinnerung ebenso formen kann wie ein vererbter Gegenstand, illustriert hingegen der Film Grbavica (2006, Esmas Geheimnis) von Jasmila Žbanić. Das fehlende Dokument über den angeblichen heldenhaften Tod des Vaters im Bosnienkrieg wirft für Esmas Tochter Fragen auf, die dazu führen, dass das Geheimnis um Saras anonyme Zeugung im Zuge der Massenvergewaltigungen in einem Lager gelüftet wird. Saras Vaterlosigkeit ist durch ein fehlendes Dokument materialisiert, das zugleich die schwere Greifbarkeit dieser verschwiegenen Episode im autobiografischen Gedächtnis symbolisiert.

Demgegenüber steht der beglaubigte Heldentod des Vaters ihres Freundes Samir, der zusätzlich zu dem entsprechenden Dokument über die Schusswaffe des Gefallenen verfügt. Das vorhandene, wie auch das nicht vorhandene Objekt werden letztendlich zu einem Trigger, der das transgenerationale Trauma symptomatisch offenlegt: Samir entwickelt eine enge Bindung an das ihm vom verlorenen Vater vererbte Objekt, das bei Konflikten seine aggressiven Gedanken anzieht. (Žbanić 2006: 1:04:35-1:05:05) Auch Saras fehlendes Objekt wird kurzfristig durch diese Waffe ersetzt, mit der sie, ebenfalls im Affekt, die Enthüllung des Familiengeheimnisses erzwingt. (ebd. S. 1:14:37-1:15:20)

Es ist bezeichnend, dass auch die Annäherung der beiden Jugendlichen über diese Waffe erfolgt. Nachdem Sara Samirs romantisches Werben nicht zulässt, kommen sich die beiden zum ersten Mal physisch sowie emotional näher, als er sie in seine mit der Waffe verbundenen Erinnerungen einbezieht und ihr erlaubt, diese mit ihm gemeinsam zu betätigen. (ebd. S. 50:20-51:35) Als wichtiges Erinnerungsobjekt ergänzt sie in beiden Biografien den jeweils fehlenden, unersetzlichen Vater, von denen einer im Krieg gefallen ist und der andere sich als Kriegsverbrecher erweist.

Aufgrund ihrer Objektbeschaffenheit vermag die Waffe die gemeinsamen Emotionen von Wut, Sehnsucht und Trauer zu bündeln, und sie erlaubt den Figuren, diesen einen kathartisch-physischen Ausdruck zu verleihen. Auf diese Weise verbindet sie diese und ihr komplementäres transgenerational weitergegebenes traumatisches Erbe. 


\section{Erzählerische Formungen der Lücke im Familiengedächtnis}

Nach der Eskalation zwischen Mutter und Tochter, bei der in Grbavica die für beide traumatisierenden Ursprünge Saras offengelegt werden, ist die Erzählung zunächst gestört. An die Stelle der bisherigen scheinbaren Kohärenz treten symptomatische Handlungen, der physisch ausgetragene Konflikt illustriert v.a. die Hilflosigkeit und Verzweiflung beider Figuren, die sie zunächst gegeneinander und danach gegen sich selbst richten. Beide benötigen Zeit, um die Bruchstücke des bisherigen autobiografischen Narrativs zu einer neuen kohärenten und zugleich lebensbejahenden Erzählung zu ordnen. ${ }^{3}$

Kokurins Tisch im gleichnamigen Roman steht, ähnlich wie in Grbavica die Waffe, für die schwerwiegende Leerstelle des durch Schweigen abgeschirmten Vaters. So könnte auch die Widmung verstanden werden, die an einen Uwe Tisch gerichtet ist. Das Buch enthält keine Figur mit einem solchen Vor- oder Nachnamen. Dass letzterer jedoch mit dem wichtigsten Objekt im Text korrespondiert, welches außerdem als Buchtitel gewählt wurde, scheint kein Zufall zu sein. Genau genommen enthält der Roman nicht eine, sondern zwei sehr ähnliche Leerstellen des Vaters: Die erste betrifft den Vater der Protagonistin; diese wird anhand des Tischs und der Erzählungen ihrer Mutter ausführlich dargestellt und geschlossen, während die zweite bestehen bleibt. Diese gilt dem unbekannten Vater ihres Sohnes und ist ebenso wichtig.

Transgenerationale Aspekte von Trauma werden in der Figurenkonstellation von Kokurins Tisch besonders deutlich, denn die Protagonistin lebt wie ihre Mutter ohne Partner. Bemerkenswert am vorliegenden Text ist außerdem die Kommunikationssituation, denn über weite Passagen hinweg gibt die Protagonistin die Jugendbiografie ihrer Mutter wieder, und zwar auf Ereignisse bezogen, die sie selbst nicht miterlebt hat. Dies ist insofern bemerkenswert, als der Roman nämlich einen konkreten Adressaten hat, und zwar ihren erwachsenen Sohn, der in der Handlung, abgesehen von seiner zweifachen Erwähnung als Adressat, ausgespart bleibt.

Du fragst, wer dein Vater ist? Glaube mir: es gibt Dinge, die man nicht wissen muss. Und keineswegs darum, weil irgendein Geheimnis dahinterstecken würde. Ich habe viel darüber nachgedacht, was eine Frau ihrem Sohn, der Antworten braucht, an ihrem Lebensabend mit auf den Weg geben kann und ich habe verstanden: ich muss dir diese Geschichte erzählen. (Kokurin 2008: 8-9)

3 Vgl. hierzu ausführlicher: Richter 2017: 173-175. 
Das Auffällige an dieser Konstellation ist vor allem, dass die Protagonistin ihrem Sohn von der Jugend ihrer Mutter berichtet, nicht jedoch von ihrer eigenen, und dass sie die von ihr selbst aufgeworfene Frage nach dem abwesenden Vater ihres Sohnes unbeantwortet belässt. Das angedeutete Spiegelverfahren einer transgenerationalen Traumaverarbeitung wird so nämlich nicht fortgesetzt, da die Mutter ihrem Sohn keine Informationen über sich selbst preisgibt, wie ihre Mutter es anhand des Tisches tat. Dass in diesem Text zwei Phantome verbleiben, nämlich die beiden Hauptfiguren - d.h. die Protagonistin und ihr Sohn, und dass die Erzählinstanz unfähig erscheint, sich zu den intendierten Themen überhaupt zu äußern, wird am Ende deutlich: „Ich hatte sehr gehofft, wenn ich es fertigbringe, dir die Geschichte unserer Familie zu erzählen, dann würde ich mich vielleicht selbst in ihr zurechtfinden ... Aber worin eigentlich? ... Alleine finde ich keine Antwort.“(ebd. 183)

Die Protagonistin steht an der Grenze zur Sprachlosigkeit, als Erzählerin ist sie diejenige, die erzählen, ordnen, bewerten, erklären sollte, allerdings ist sie dazu selbst nicht in der Lage, weshalb sie eine Geschichte wiedergibt, die ihr selbst erzählt und daher bereits in Worte gefasst wurde. Das transgenerationale Trauma wird hier ebenfalls thematisiert.

Ich neige immer stärker zu der Auffassung, dass die Jahre vergehen, der Mensch sich im Innersten aber nicht ändert. Als lenke ein unsichtbarer Magnet ihn auf die immer gleiche Umlaufbahn, auf alte Lieben und alte Fehler. Und es bedarf einer beträchtlichen Anstrengung, einer unvorstellbaren seelischen Erschütterung, vergleichbar mit derjenigen, die ich erlebt habe, als ich mit meiner Mutter und unserem Tisch zu Fuß unterwegs war, um versteinerte Denkweisen zu zertrümmern und womöglich neuen Halt zu finden. Vielleicht... Ob meine Geschichte bei dir eine solche Erschütterung bewirkt - dessen bin ich mir nicht sicher. Aber ich wünschte es so sehr... (ebd. 184)

Was haben die zuvor erwähnten erzählerischen Auffälligkeiten nun zu bedeuten eine Erzählerin, die nicht erzählen kann und ein fiktiver Adressat, der als Individuum nicht greifbar wird? ${ }^{4}$ Da unklar bleibt, ob dieses Manuskript letztendlich übergeben wird, oder nicht, wäre eine mögliche Interpretation, dass die Protagonistin den Text primär für sich selbst formuliert, und dass der Kontext ihres Soh-

\footnotetext{
4 Franz Breuer thematisiert aus psychologischer Sicht die Schwierigkeit, eine geeignete Situation für die Weitergabe von Erbstücken und den damit verbundenen Erinnerungen zu finden, welche dieser Konstellation im Roman entspricht: „Mitunter neigt ein (Vor-)besitzer zum Festhalten an der Objektfügung, und zum Hinausschieben des Zeitpunkts der Weitergabe [...]. Und schließlich werden die Bedürfnisse und die Lebensplanungsumstände einer möglichen Nachfolgeperson auf diese Weise nicht berücksichtigt. [...] Der ,richtige Zeitpunkt‘ für eine Übergabe wird bei so einem Vorgehen möglicherweise verpasst.“ (Breuer 2015: 38)
} 
nes ihr lediglich einen imaginativen Rahmen für das Erzählen bietet. Vom Inhalt ausgehend verhält sich die Erzählsituation nämlich genau umgekehrt, als durch die formalen Erzählinstanzen festgelegt: Die Protagonistin versucht aus ihrer eigenen Mutterrolle heraus, jene Geschichte $\mathrm{zu}$ imaginieren und/oder $\mathrm{zu}$ formulieren, die zu hören sie sich von ihrer eigenen Mutter gewünscht hätte.

Als der Roman nämlich im Moskauer Hotel endet, wird deutlich, dass die eigentliche Suche des Texts einem in der Realität gescheiterten Verstehen der eigenen unzugänglichen Mutter galt: „Meine Mutter fuhr mit den Fingern unruhig über die Perlen. Sie war vollkommen verändert. Jener Mensch, der aus ihrem verborgenen Inneren zutage getreten war, meine Mutter, von der ich mein Leben lang sehnsüchtig geträumt und sie endlich gefunden hatte - war verschwunden.“ (Kokurin 2008: 197)

\section{Objekt, Weitergabe, physische Konfrontation und Erzählen}

Transgenerational weitergegebene Objekte füllen in den behandelten Texten jeweils den Bewusstseinsraum für etwas Unaussprechliches, das meist mit traumatischen Einschnitten in der Familiengeschichte in Zusammenhang steht. Viele Beispiele handeln davon, wie verschwiegene Ereignisse durch ihre symbolischindexikalische Präsenz einen Redeanlass erhalten und daraufhin über Umwege erzählt werden können. Die Besonderheit der Objekte liegt jedoch zugleich gerade darin, dass eine Versprachlichung nicht mehr unbedingt notwendig ist, da die Objekte eine Ausdrucksform anbieten, die geeignet erscheint, an die Stelle des Erzählens zu treten.

Die Weitergabe von Familienerfahrung und das ,Erzählen“ der Objekte stehen jeweils in unmittelbarem Zusammenhang mit deren Materialität: Während die Objektübergabe häufig innerhalb von Situationen stattfindet, die Qualität und Implikationen der Familienbande erahnen lassen, sind die Objekte selbst oft symbolisch aufgeladen. Ihre physikalisch-materielle Beschaffenheit und die ihnen durch ihren Gebrauch zugewiesene Verortung zum Menschen führen zu Eindrücken wie Schwere, Raumeinnahme, Nähe, Berührung, Glanz, Haltbarkeit oder Vergänglichkeit. Darüber hinaus sind diese an sich neutralen Eigenschaften mit vagen Ahnungen über ihre transgenerationale Bedeutung verbunden: mit Assoziationen wie Unheil oder Schutz, Verbundenheit oder Belastung.

Diese subjektiven Zuschreibungen gehen oft indexikalisch auf mit ihnen assoziierte Ereignisse oder die Beziehung zu Vorbesitzer*innen zurück, wofür sie sich jeweils als Symbol verfestigen. In anderen Fällen beruhen sie auch auf einer 
quasi synästhetischen Interpretation ihrer materiellen Beschaffenheit als Symbolisierung der gegenwärtigen Verortung ihrer Besitzerinnen in den Lebensumständen. Unabhängig von der Art der Symbolbildung wird das Objekt zu einem emotional behafteten Gegenstand, der diese mit ihrer Vergangenheit und deren Implikationen für die Gegenwart konfrontiert.

Im Umgang mit diesen Objekten bleibt die Art der Konfrontation jeweils den menschlichen Akteur^innen überlassen, und das Resultat solcher Auseinandersetzungen mit ihnen scheint stets ungewiss. In vorsichtig abwägenden, affektgeleiteten oder zufälligen Handlungen illustrieren die subjektiven Symbolbedeutungen eines Gegenstands jedoch scheinbar Schlüsselmomente wie Retraumatisierung, Weitergabe, Wiederholung, die Verarbeitung und das Ausweichen, Überschreiben oder Vergessen von Erfahrungen früherer Generationen.

\section{Bibliografie}

Aleschkowski, Pjotr. 1998. Der Erbe. Aus dem Russischen von Alfred Frank. Frankfurt am Main: Suhrkamp.

Aleškovskij, Petr. 1997. Vladimir Čigrincev. Moskva: Vagrius.

Assmann, Aleida. 2003. Das Gedächtnis der Dinge. In Alexandra Reininghaus (ed.), Recollecting. Raub und Restitution, 143-150. Wien: Passagen-Verlag.

Assmann, Aleida. $2010^{5}$. Erinnerungsräume. Formen und Wandlungen des kulturellen Gedächtnisses. Fünfte, durchgesehene Auflage. München: C. H. Beck.

Basseler, Michael \& Dorothee Birke. 2005. Mimesis des Erinnerns. In Astrid Erll \& Ansgar Nünning (eds.), Gedächtniskonzepte der Literaturwissenschaft. Theoretische Grundlegung und Anwendungsperspektiven (= Medien und kulturelle Erinnerung, 2), 123-147 Berlin \& New York: De Gruyter.

Bojaschow, Ilya. 2018. Muris Weg. Aus dem Russischen von Ruth Altenhofer. Klagenfurt: Drava. Bojašov, Il'ja. 2011. Put' Muri. Sankt Peterburg: Limbus Press.

Borchert, Wolfgang. 1999. Die Küchenuhr. In Ders., Draußen vor der Tür, 103-105. Hamburg: Rowohlt.

Breuer, Franz. 2015. Hybride Objekte verbinden Personen und Generationen. Weitergabe Gedenken - Transzendenz. In Günter Mey (ed.), Von Generation zu Generation. Sozial- und kulturwissenschaftliche Analysen zu Transgenerationalität, 25-53. Gießen: PsychosozialVerlag.

Dovlatov. Sergej. 2010. Čemodan. In Ders., Sobranie sočinenij v četyrech tomach. Sost. A.A. Ar'ev. T. 3, 345-482. Sankt Peterburg: Azbuka.

Eichenberg, Ariane. 2009. Familie - Ich - Nation. Narrative Analysen zeitgenössischer Generationenromane. Göttingen: Vandenhoeck Rupprecht.

Freud, Sigmund. 2015. Die Freudsche psychoanalytische Methode. In Ders., Gesammelte Werke. Bd. 1, 177-231. Altenmünster: S. Fischer.

Goldstein, Bruce E. $2015^{9}$. Wahrnehmungspsychologie. Der Grundkurs. Deutsche Ausgabe herausgegeben von Karl R. Gegenfurtner. Aus dem Englischen übersetzt von Katharina Neuser-von Oettingen und Guido Plata. Berlin \& Heidelberg: Springer. 
Hirsch, Marianne. 2012. The Generation of postmemory. Writing and visual culture after the Holocaust. New York \& Chichester: Columbia University Press.

Hirsch, Marianne \& Leo Spitzer. 2012. Testimonial Objects. In Marianne Hirsch, The Generation of postmemory. Writing and visual culture after the Holocaust, 177-199. New York \& Chichester: Columbia University Press.

Jandl, Ingeborg. 2019a. Textimmanente Wahrnehmung bei Gajto Gazdanov. Sinne und Emotion als motivische und strukturelle Schnittstelle zwischen Subjekt und Weltbild (= Slavische Literaturen, 52). Berlin.

Jandl, Ingeborg. 2019b. Kriegskindheiten in Sarajevo. In Aphorismen, Objekten und Bildern. In Gernot Howanitz \& Ingeborg Jandl (eds.), Ich-Splitter. (Cross-)Mediale Selbstentwürfe in den Slawischen Kulturen (= Wiener Slawistischer Almanach; Sonderband 96), 297-331. Frankfurt am Main et al.: Lang.

Kiš, Danilo. 2006. Sabrana dela Danila Kiša. Knj. 2: Psalam 44. Beograd: Prosveta.

Kiš, Danilo. 2019. Psalm 44. Aus dem Serbokroatischen von Katharina Wolf-Grießhaber. München: Hanser.

Kokurin, Ananij. 2018. Der Tisch. Hamburg: Osburg.

Lebedev, Sergej. 2018. Gus' Fric. Moskva: Vremja.

Lebedew, Sergej. 2018. Kronos' Kinder. Frankfurt am Main: S. Fischer.

Levine, Peter. $2016^{2}$. Trauma und Gedächtnis. Die Spuren unserer Erinnerung in Körper und Gehirn. Wie wir traumatische Erfahrungen verstehen und verarbeiten. Aus dem amerikanischen Englisch von Silvia Autenrieth. München: Kösel-Verlag.

Richter, Angela. 2017. Vergewaltigung in Literatur und Film über den Bosnienkrieg. Zur Darstellung traumatischer Grenzerfahrung. In Gabriela Lehmann-Carli et al. (eds.), Zerreißproben: Trauma - Tabu - EmpathieHürden (= Ost-West-Express. Tabu und Übersetzung, 27), 155-177. Berlin: Frank \& Timme.

Volc, D., Tilscher, H. \& M. Hanna. 1988. Halswirbelsäule und Psychosomatik. Diagnostik und therapeutische Aspekte. In Hans Tilscher et al. (eds.), Kopfschmerzen. Zur Diagnostik und Therapie von Schmerzformen außer Migräne, 75-77. Berlin: Springer.

Žbanić, Jasmila. 2006. Grbavica. Sarajevo, Karlsruhe, Zagreb: Deblokada, noirfilm, Jadran. 